\title{
GilBERT: Generative Vision-Language Pre-Training for Image-Text Retrieval
}

\author{
Weixiang Hong, Kaixiang Ji, Jiajia Liu, Jian Wang, Jingdong Chen, Wei Chu \\ Ant Group \\ \{hwx229374,kaixiang.jkx,lekun.ljj,bobblair.wj,jingdongchen.cjd,weichu.cw\}@antgroup.com
}

\begin{abstract}
Given a text/image query, image-text retrieval aims to find the relevant items in the database. Recently, visual-linguistic pre-training (VLP) methods have demonstrated promising accuracy on imagetext retrieval and other visual-linguistic tasks. These VLP methods are typically pre-trained on a large amount of image-text pairs, then fine-tuned on various downstream tasks. Nevertheless, due to the natural modality incompleteness in image-text retrieval, i.e., the query is either image or text rather than an image-text pair, the naive application of VLP to image-text retrieval results in significant inefficiency. Moreover, existing VLP methods cannot extract comparable representations for a single-modal query and multi-modal database items. In this work, we propose a generative visual-linguistic pre-training approach, termed as GilBERT, to simultaneously learn generic representations of image-text data and complete the missing modality for incomplete pairs. In testing phase, the proposed GilBERT facilitates efficient vector-based retrieval by providing unified feature embedding for query and database items. Moreover, the generative training not only makes GilBERT compatible with non-parallel text/image corpus, but also enables GilBERT to model the image-text relationships without suffering massive randomly-sampled negative samples, leading to superior experimental performances. Extensive experiments demonstrate the advantages of GilBERT in image-text retrieval, in terms of both efficiency and accuracy.
\end{abstract}

\section{CCS CONCEPTS}

- Information systems $\rightarrow$ Multimedia and multimodal retrieval; Similarity measures.

\section{KEYWORDS}

Image-text retrieval; Visual-linguistic pre-training.

\section{ACM Reference Format:}

Weixiang Hong, Kaixiang Ji, Jiajia Liu, Jian Wang, Jingdong Chen, Wei Chu. 2021. GilBERT: Generative Vision-Language Pre-Training for Image-Text Retrieval. In Proceedings of the 44th International ACM SIGIR Conference on Research and Development in Information Retrieval (SIGIR '21), fuly 11-15, 2021, Virtual Event, Canada. ACM, New York, NY, USA, 10 pages. https: //doi.org/10.1145/3404835.3462838

Permission to make digital or hard copies of all or part of this work for personal or classroom use is granted without fee provided that copies are not made or distributed for profit or commercial advantage and that copies bear this notice and the full citation on the first page. Copyrights for components of this work owned by others than ACM must be honored. Abstracting with credit is permitted. To copy otherwise, or republish, to post on servers or to redistribute to lists, requires prior specific permission and/or a fee. Request permissions from permissions@acm.org.

SIGIR '21, July 11-15, 2021, Virtual Event, Canada

(C) 2021 Association for Computing Machinery.

ACM ISBN 978-1-4503-8037-9/21/07 . \$15.00

https://doi.org/10.1145/3404835.3462838
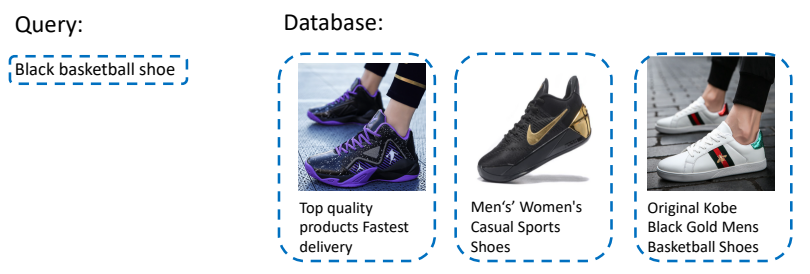

Figure 1: In retrieval applications like E-commence product search, it is common that the user-inputted query is purely text, while most of the database items are image-text pairs. Existing VLP methods are incapable to extract comparable and comprehensive features for query and database items.

\section{INTRODUCTION}

The success of retrieval heavily depends on representations. On the one hand, high-quality representations enable accurate distance computation and distance-based ranking, leading to desirable retrieval results. On the other hand, due to the explosive growth of modern data, the representations are expected to be convenient in storage and efficient in mutual distance computation. The requirements on storage and efficiency make vector to be a suitable representation form for original data. Compared with model-based similarity inference, the vector-based distance computation (VBDC) is significantly faster, e.g., inner product, Euclidean distance, etc. Moreover, vectors can be hashed [21], quantized [16] or indexed [3] to further optimize the storage and computation cost.

We study image-text retrieval in this work. To be more specific, we group image-text retrieval into two categories: cross-modal retrieval and multi-modal retrieval, depending on whether the database items are single-modal text/image or image-text pairs. In details, cross-modal retrieval performs retrieval across different modalities (e.g., texts v.s. images). It takes one type of data as the query to retrieve relevant items of another type. Compared with cross-modal retrieval, multi-modal retrieval is relatively less concerned, but we argue that it is worth studying due to its great value in industrial applications. Take Figure 1 as an example of multi-modal retrieval, the user-inputted query is purely text for E-commerce product search, while most of the database items are image-text pairs. Extracting comparable and comprehensive features containing multi-modal knowledge for both the query and database items is a more challenging problem under such an asymmetrical setting. On the one head, it is naturally more comprehensive if the representations of database items are extracted from image-text pairs rather than a single modal, on the other hand, it is also a must that the feature representations of the query (image or text) and database items (image-text pairs) are comparable. 
Recently, visual-linguistic pre-training (VLP) is proposed for tasks at the intersection of computer vision (CV) and natural language processing (NLP), such as image-text retrieval, image captioning, visual question answering, etc. VLP generally takes language embeddings and visual region features as input, and relies on the self-attention mechanism of Bidirectional Transformers (BERT) [9] to learn generic representations from massive image-text pairs $[28,30,34,36,47,51,68]$. Following the "pre-train \& fine-tune" pipeline $[42,54]$ in CV and NLP, VLP can effectively transfer the knowledge learned in pre-training to downstream visual-linguistic tasks and obtain state-of-the-art results on several applications. Take cross-modal retrieval on COCO dataset as example, the Recall@1 of Oscar [34] is higher than existing traditional cross-modal retrieval methods like SCG [45] and PFAN [57] by at least 10 points in both image and text retrieval tasks.

Unfortunately, though the existing VLP methods demonstrate promising cross-modal retrieval accuracy, they are incapable to generate comparable vector representations for query and database items. Thus, they cannot well tackle multi-modal retrieval task, which has wide applications in E-commence product search, image search by image, etc. Moreover, in the inference stage, current VLP methods have to exhaustively pair the query and each database item, then feed the pairs to the VLP model for predicting matching scores. It is computation-intensive and time-consuming to perform modelbased similarity inference compared with VBDC. The inefficiency of VLP methods hinders their application to practical retrieval scenarios despite their promising accuracy.

To address the problems above, we propose a generative visuallinguistic BERT (shorted as GilBERT) for tackling image-text retrieval problem. Specifically, we harness visual-linguistic transformers to learn comprehensive representations of image-text pairs, meanwhile train generative models to perform image-to-text captioning and text-to-image synthesizing for incomplete pairs. We integrate captioning and synthesizing models with transformers and train them in an end-to-end manner. In the testing phase, the trained generative model can complete the missing modality based on the available one, i.e., text-to-image synthesizing for text input and image-to-text captioning for image input. The completed image-text pairs are further fed to the visual-linguistic transformers, so that a unified vector representation can be extracted for performing multi-modal retrieval. Moreover, existing VLP methods often model the relationship between image and text modals by utilizing a Image-Text Alignment loss, which introduces massive negative samples into training [47]. We find by experiments that the introduced negative samples harm the retrieval performances. In contrast, thanks to the generative modeling, the proposed GilBERT does not suffer from the massive negative samples caused by ImageText Alignment, hence further boost the retrieval accuracy upon existing VLP works. Extensive experiments demonstrate the efficacy and efficiency advantages of our proposed GilBERT. The contributions of this paper are summarized below:

- We integrate visual-linguistic transformers with generative models, to tackle the inefficiency of existing VLP methods in retrieval task. The proposed GilBERT runs significantly faster than existing VLP methods such as $[28,34]$ in retrieval efficiency, i.e., GilBERT takes 0.9 seconds v.s. 12 minutes by [34] in COCO $5 \mathrm{~K}$ testset.

- We find that the Image-Text Alignment loss in existing VLP works hurts the retrieval performances since it introduces massive negative samples into training [47]. By harnessing generative modeling, the Image-Text Alignment loss is transformed into a part of the adversarial loss, which prevents the Image-Text Alignment loss from involving massive negative samples in the training progress. As a result, our GilBERT further boosts the retrieval accuracy of existing VLP works.

- We also achieve improved efficiency in data utilization. As shown in Section 4.2, the GilBERT models trained with additional non-parallel image and text corpus consistently outperform those trained only with parallel image-text pairs. Given that paired data is costly to collect [31], the compatibility to incomplete data makes it possible to further improve our GilBERT by augmenting the training set with additional non-parallel image and text corpus.

\section{RELATED WORK}

Humans perceive the world through many channels. Given that any individual channel might be incomplete or noisy, the representation learned from multi-modal data is relatively more comprehensive. In this section, we firstly review visual-linguistic pre-training (VLP), which is the state-of-the-art solution for learning generic representations from $\mathrm{V}+\mathrm{L}$ data, then we discuss image-text retrieval, which is a challenging down-stream task that existing VLP methods do not work well at.

\subsection{Visual-Linguist Pre-Training}

Learning generic representations is fundamental to a wide range of machine learning tasks. In computer vision, backbone networks designed for and pre-trained on ImageNet [8] classification was found to be effective for numerous image recognition applications. Meanwhile, Bidirectional Transformer (BERT) [9] pre-trained with unsupervised objectives on large language corpus excelled at a variety of downstream natural language processing tasks.

Motivated by the success of pre-training in CV and NLP, visionlanguage pre-training (VLP) was proposed for tasks at the intersection of vision and language. Existing VLP methods rely on the self-attention mechanism of Transformers [52] to learn joint representations which are appropriately contextualized in both modalities. They employed BERT-like objectives [52] to learn cross-modal representations from a concatenated sequence of visual region features and language token embeddings. The recently published Oscar [34], which is the state-of-the-art method, extended visual region features and language token embeddings by adding object tags so as to improve the alignment between image and text modalities. Please refer to Table 1 for a complete comparison of GilBERT and existing VLP methods.

\section{$2.2 \quad$ Image-text Retrieval}

We divide image-text retrieval into cross-modal retrieval and multimodal retrieval, depending on whether database items are singlemodal images/texts or multi-modal image-text pairs. 


\begin{tabular}{|c|c|c|c|c|c|}
\hline Method & Architecture & Visual Tokens & Pre-train Datasets & Pre-train Tasks & Downstream Tasks \\
\hline VideoBERT [50] & single cross-modal Transformer & video frame & Cooking $312 \mathrm{~K}[50]$ & $\begin{array}{l}\text { 1) sentence-image alignment } \\
\text { 2) masked language modeling } \\
\text { 3) masked visual-words prediction }\end{array}$ & $\begin{array}{l}\text { 1) zero-shot action classification } \\
\text { 2) video captioning }\end{array}$ \\
\hline CBT [49] & $\begin{array}{l}\text { two single-modal Transformer } \\
\text { (vision \& language respectively) } \\
+ \text { one cross-modal Transformer }\end{array}$ & video frame & Cooking $312 \mathrm{~K}[50]$ & $\begin{array}{l}\text { 1) sentence-image alignment } \\
\text { 2) masked language modeling } \\
\text { 3) masked visual-feature regression }\end{array}$ & $\begin{array}{l}\text { 1) action anticipation } \\
\text { 2) video captioning }\end{array}$ \\
\hline ViLBERT [36] & $\begin{array}{l}\text { one single-modal Transformer } \\
\text { (language) } \\
\text { + one cross-modal Transformer } \\
\text { (with restricted attention pattern) }\end{array}$ & image RoI & Conceptual Captions [44] & $\begin{array}{l}\text { 1) sentence-image alignment } \\
\text { 2) masked language modeling } \\
\text { 3) masked visual-feature classification }\end{array}$ & $\begin{array}{l}\text { 1) visual question answering } \\
\text { 2) visual commonsense reasoning } \\
\text { 3) grounding referring expressions } \\
\text { 4) image retrieval } \\
\text { 5) zero-shot image retrieval }\end{array}$ \\
\hline $\mathrm{B} 2 \mathrm{~T} 2[2]$ & single cross-modal Transformer & image RoI & Conceptual Captions [44] & $\begin{array}{l}\text { 1) sentence-image alignment } \\
\text { 2) masked language modeling }\end{array}$ & 1) visual commonsense reasoning \\
\hline LXMERT [51] & $\begin{array}{l}\text { two single-modal Transformer } \\
\text { (vision \& language respectively) } \\
\text { + one cross-modal Transformer }\end{array}$ & image RoI & $\begin{array}{l}\text { COCO Caption [4] } \\
+ \text { VG Caption } \\
+ \text { VG QA } \\
+ \text { VQA } \\
+ \text { GQA [23] }\end{array}$ & $\begin{array}{l}\text { 1) sentence-image alignment } \\
\text { 2) masked language modeling } \\
\text { 3) masked visual-feature classification } \\
\text { 4) masked visual-feature regression } \\
\text { 5) visual question answering }\end{array}$ & $\begin{array}{l}\text { 1) visual question answering } \\
\text { 2) natural language visual reasoning }\end{array}$ \\
\hline VisualBERT [30] & single cross-modal Transformer & image RoI & COcO Caption [4] & $\begin{array}{l}\text { 1) sentence-image alignment } \\
\text { 2) masked language modeling }\end{array}$ & $\begin{array}{l}\text { 1) visual question answering } \\
\text { 2) visual commonsense reasoning } \\
\text { 3) natural language visual reasoning } \\
\text { 4) grounding phrases }\end{array}$ \\
\hline Unicoder-VL [28] & single cross-modal Transformer & image RoI & Conceptual Captions [44] & $\begin{array}{l}\text { 1) sentence-image alignment } \\
\text { 2) masked language modeling } \\
\text { 3) masked visual-feature classification }\end{array}$ & $\begin{array}{l}\text { 1) image-text retrieval } \\
\text { 2) zero-shot image-text retrieval }\end{array}$ \\
\hline VL-BERT [47] & single cross-modal Transformer & image RoI & $\begin{array}{l}\text { Conceptual Captions [44] } \\
+ \text { BooksCorpus } \\
+ \text { English Wikipedia }\end{array}$ & $\begin{array}{l}\text { 1) masked language modeling } \\
\text { 2) masked visual-feature classification }\end{array}$ & $\begin{array}{l}\text { 1) visual question answering } \\
\text { 2) visual commonsense reasoning } \\
\text { 3) grounding referring expressions }\end{array}$ \\
\hline Oscar [34] & single cross-modal Transformer & $\begin{array}{l}\text { image RoI } \\
+ \text { object tags }\end{array}$ & $\begin{array}{l}\text { Conceptual Captions [44] } \\
+ \text { COCO [35] } \\
+ \text { SBU Captions [39] } \\
+ \text { flicker30k [60] } \\
+ \text { GQA [23] }\end{array}$ & $\begin{array}{l}\text { 1) sentence-image alignment } \\
\text { 2) masked language modeling } \\
\text { 3) masked visual-feature classification }\end{array}$ & $\begin{array}{l}\text { 1) image-text retrieval } \\
\text { 2) image captioning } \\
\text { 3) novel object captioning } \\
\text { 4) visual question answering } \\
\text { 5) visual commonsense reasoning } \\
\text { 6) GQA } \\
\text { 7) natural language visual reasoning }\end{array}$ \\
\hline GilBERT & $\begin{array}{l}\text { single cross-modal Transformer } \\
+ \text { two cross-modal generator }\end{array}$ & image RoI & $\begin{array}{l}\text { Conceptual Captions [44] } \\
+ \text { COCO [35] } \\
+ \text { SBU Captions [39] } \\
+ \text { flicker30k [60] } \\
+ \text { GQA [23] }\end{array}$ & $\begin{array}{l}\text { 1) masked language modeling } \\
\text { 2) masked visual-feature classification }\end{array}$ & $\begin{array}{l}\text { 1) image-text retrieval } \\
\text { 2) image captioning } \\
\text { 3) novel object captioning } \\
\text { 4) visual question answering } \\
\text { 5) natural language visual reasoning }\end{array}$ \\
\hline
\end{tabular}

Table 1: Comparisons with existing VLP methods.

Cross-modal retrieval is traditionally tackled by injective embedding methods $[10,12,24,27,29,45,57,58,62-65,68]$ that seek a shared feature space between both modalities. The features of text/image are extracted by a modality-specific encoder, then aligned by correlation maximization, ranking loss, mutual generation, etc. After learning is done, the features of database items are computed and indexed. When a new query arrives, the corresponding encoder is called to extract the feature for efficient VBDC.

Recently, VLP methods demonstrate advantageous retrieval accuracy. For example, in COCO retrieval, the state-of-the-art injective embedding method obtains Recall@1 of Image Retrieval at $~ 62$, while Oscar [46] boosts it up to 75.7 with the aid of pre-training. However, the lack of vector representations hinders the application of VLP methods to industry applications. Though some two-stream VLP methods like $[36,37]$ can partially reduce computation in inference stage by pre-computing features of database items, their retrieval performances are hurt due to insufficient interactions between image and text modalities (See Table 2). Moreover, these two-stream VLP methods [36, 37] still fail to extract a valid representation for the query and do not support vector-based retrieval.

Perhaps GXN [12] shares the most similar idea with our work, i.e., retrieval by generation, however, 1). GXN mainly focus on cross-modal retrieval, and is incapable to extract a unified representation for image-text pair. 2). GXN does not support large-scale pre-training on massive image-text pairs, hence it cannot transfer knowledge from other domains to retrieval tasks.

Multi-modal retrieval aims to perform retrieval in an asymmetrical setting, where the query is single-modal text/image and database items are mostly image-text pairs. A naïve solution is to ignore either the text or image modalities of database items, and degrade the problem into cross-modal retrieval or purely image/text retrieval. Nevertheless, different modalities sometimes have complementary information, and comprehensive representations extracted from multi-modal data are expected to demonstrate better performances in retrieval. As we shown by experiments, the proposed GilBERT offers unified representations of image/text and image-text pairs, leading to advantageous retrieval accuracy.

\section{METHOD}

We present the proposed GilBERT in this section. Starting with a revisit to BERT model [9] in Section 3.1, we elaborate on the architecture of GilBERT in Section 3.2 to Section 3.4. Then, we introduce the training details in Section 3.5. 


\subsection{Revisiting BERT Model}

The input to BERT [9] is a set of word embeddings. They are processed by a multi-layer transformer [52], where the embedding of each element is transformed by aggregating features from the other elements with adaptive attention weights. We refer the readers to [52] for more details of transformers.

In BERT pre-training, the Masked Language Modeling task is introduced. The embedding of a certain input word is randomly masked out, with its token embedding marked by a special [MASK] token. The BERT model is trained to predict the masked word from linguistic clues of all the other unmasked elements. The second pre-training task, Next Sentence Prediction, focuses on modeling the relationship between sentences, i.e. two sentences are sampled from the input document, and a classifier is appended to predict whether the second sentence is the direct successor of the first.

\subsection{GilBERT}

Figure 2 shows the overall architecture for the proposed generative visual-linguistic pre-training framework, which consists of three main components: visual-linguistic feature embedding (the blue path), image-to-text captioning (the yellow path), and text-to-image synthesizing (the green path). We build our transformer for visuallinguistic feature embedding upon BERT [52], with two components changed:

Visual tokens. We treat visual regional features as tokens for transformers, and mark them by an additional segment embedding. The visual regional features are obtained by applying Faster R-CNN [42] to the input image. Specifically, we concatenate the region feature vector (2048-d) and region position vector (4-d) to form a positionsensitive visual feature vector, which is further transformed by a linear projection to ensure that it has the same vector dimension as the word embedding vector.

Losses. We train our GilBERT with Masked Token Modeling and Image Text Matching loss. For Masked Token Modeling, we randomly mask text or visual token by a special token [MASK], and train the model to predict the masked word (for text token) or the masked region class (for visual token). Let $x=\left\{x_{1}, \ldots, x_{N}\right\}$ be the a input sample consisting of $N$ embedded token features, the Masked Token Modeling loss can be formulated as:

$$
\log P(x \mid \theta)=\frac{1}{Z(\theta)} \sum_{i=1}^{N} \log \phi_{i}(x ; \theta)
$$

where $\phi_{i}(x ; \theta)$ is the potential function with parameter $\theta$, and $Z(\theta)$ is the partition function.

For Image Text Matching, we train a binary classifier to predict whether the pair is a faked pair produced by our generative model or not. Given the input pair $x$, let $t \in\{0,1\}$ indicate whether one of its modalities is missing, the loss function is defined as:

$$
L=-E_{(x, t)}\left[t \log \left(g\left(x^{L}\right)\right)+(1-t) \log \left(1-g\left(x^{L}\right)\right)\right]
$$

where $x^{L}$ is the final output feature of the [CLS] element (at the $L$-th layer), and $g\left(x^{L}\right)$ is the classifier output. $E_{(x, t)}$ is the expectation over all $(x, t)$ in training set. It is worth noting that existing VLP methods generally compute Image Text Alignment over randomly sampled negative pairs from the training set, while the proposed

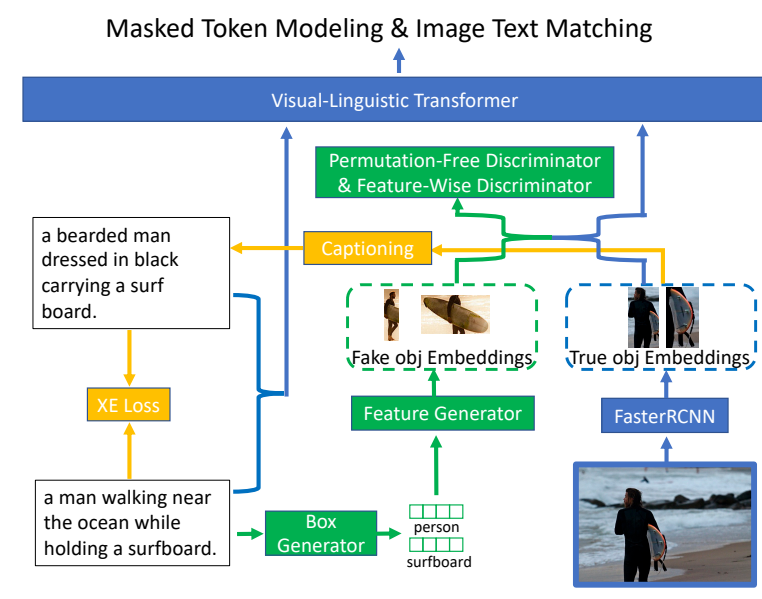

Figure 2: The text-to-image synthesizing, image-to-text captioning, and visual-linguistic transformers are illustrated in different colors. (a). Marked in blue, Faster R-CNN [42] are utilized to extract objects feature embedding from the input image, which is paired with input text and fed to the visuallinguistic transformers. (b). The yellow part demonstrates the image-to-text captioning process, where XE Loss stands for cross-entropy loss. (c). The green text-to-image synthesizing path consists of box generation, feature synthesizing, and discriminators.

Image Text Matching loss are computed on the fake image-text pairs from the generative model.

\subsection{Image-to-Text Generation}

For the image-to-text captioning (yellow path in Figure 2), our goal is to train a captioner that generates sentences similar to the ground-truth captions. In particular, we use Faster R-CNN [42] to obtain image representation $v$ by considering the entire input image as a proposal, then decode the image representation into a sentence with a recurrent neural network (RNN) [54]. We train our model with a cross-entropy (XE) loss defined as:

$$
\mathcal{L}_{X E}=-\sum_{t=0}^{T-1} \log p\left(w_{t} \mid w_{0: t-1}, v ; \theta_{t}\right)
$$

where $w_{t}$ is the ground-truth word, $p\left(w_{t} \mid w_{0: t-1}, v ; \theta_{t}\right)$ is the output probability of word $w_{t}$ given by the decoder with parameter $\theta$.

We found by experimentation that RNN-based solution achieved satisfactory performances in our tasks. Nevertheless, we conjecture that GilBERT is compatible with other powerful image captioning methods such as those based on LSTM [13] or reinforcement learning [43].

\subsection{Text-to-Image Synthesizing}

Compared with image-to-text captioning in Section 3.3, text-toimage synthesizing, which is commonly tackled by GAN conditioned on input text $[38,41]$, is a more challenging task for three reasons: 1). In general, text-to-image synthesizing suffers from the highly multi-modal distribution [12,32] of images conditioned on 
a text representation. 2). The goal of our generator is to synthesize object features as visual tokens for transformers. Therefore, unlike most existing text-to-image synthesizing works that produce only a single image [6,67], our generative model is expected to generate a set of location-aware objects features; 3 ). Even if a set of objects feature is generated, the discriminator needs to be permutation invariant for an orderless set input. In this section, we propose a two-stage object feature generator and a novel permutation-free discriminator to address the concerns above.

3.4.1 Object Feature Generator. As illustrated in Figure 2, we decompose the text-to-image synthesizing process into two stages: a box generator firstly produces a sequence of bounding boxes, then a feature generator synthesizes their feature embedding conditioned on the generated boxes.

Box generation. We train an attentive seq2seq [14] model as the box generator:

$$
B_{1: T}=\left[B_{1}, B_{2}, \ldots, B_{T}\right] \sim G_{b o x}(e),
$$

where $e$ is the embedded word vector, $B_{t}=\left(b_{t}, l_{t}\right)$ is a tuple of the bounding box of $t$ 's object and its class label. The box generator is trained by minimizing the negative log-likelihood with groundtruth bounding boxes. In testing time, we terminate the sampling when the sampled class label corresponds to a special termination indicator class, i.e., the number of objects is conditioned on the text. Feature synthesizing. Given the bounding boxes $B_{1: T}$, the feature generator predicts the corresponding feature embedding of each bounding box, i.e.,

$$
\hat{f_{1: T}}=G_{\text {feature }}\left(B_{1: T}, z_{1: T}\right)
$$

where $z_{t} \sim \mathcal{N}(0,1)$ is a random noise vector. Since the generated features not only need to match the location and category information provided by $B_{1: T}$, but also should be aligned with its surrounding context, we build $G_{\text {feature }}$ based on a bi-directional convolutional LSTM $[14,59]$ for realistic feature synthesizing.

3.4.2 Discriminator. Our discriminators consists of three parts: Permutation free discriminator. In order to make a model invariant to input permutation, three strategies exist: 1) sort input into a canonical order; 2) treat the input as a sequence to train an RNN, but augment the training data by all kinds of permutations; 3 ) use a simple symmetric function to aggregate the information from each point. Here, a symmetric function takes $n$ vectors as input and outputs a new vector that is invariant to the input order. For example, + and $*$ operators are symmetric binary functions.

As discussed in [40], a canonical sorting does not exist in highdimensional space (the visual features are 2048-d), and the order of input matters for RNN [53], thus we build our permutation-free set discriminator by applying symmetric functions on elements in the set, as illustrated in Figure 3. All operators in our permutation free discriminator are invariant to the order of inputs.

Feature-wise discriminator. Besides, we employ a feature-wise discriminator that takes a single object feature as input and predicts whether it is true or fake. The feature-wise discriminator and permutation-free discriminator work together to achieve better synthesizing quality, with different focuses on local feature-level generation and global image-level generation, respectively.

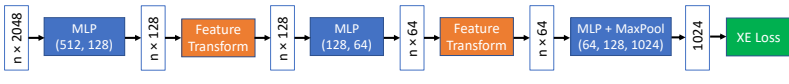

(a) Permutation free discriminator.

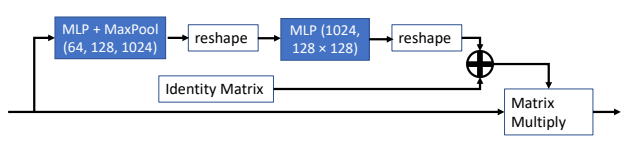

(b) Feature Transform module.

Figure 3: Details of the proposed permutation free discriminator for set input. (a). The permutation free discriminator mainly consists of MLP and Feature Transform module. MLP stands for Multi-Layer Perceptron, with numbers in the bracket as layer sizes. (b). The Feature Transform module predicts a square matrix, which is multiplied to the input for transformation. The output of Feature Transform module is initialized by an identity matrix.

Conditional discriminator. The permutation-free discriminator and feature-wise discriminator presented above are unconditional to text, thus, an additional discriminator is required to assess whether the generated visual features agree with the text or not. We note that the Image Text Matching loss in Section 3.2 essentially plays the role of the conditional discriminator [19]. The three kinds of discrimination jointly provide thorough supervision to the textto-image synthesizing process, leading to promising experimental results as shown in Section 4.3.2.

\subsection{Optimization}

3.5.1 Pre-training. We do not update the generative components of our GilBERT during pre-training, only the visual-linguistic transformer is trained by Masked Token Modeling. We note that most existing visual-linguistic pre-training methods utilize an additional Image-Text Alignment loss, i.e., a cross-entropy loss on the negative pairs that are randomly sampled from the training set, while VL-BERT [47] and the proposed GilBERT are only pre-trained with Masked Visual/Linguistic Token Loss. We use AdamW optimizer to train GilBERT $_{\mathrm{B}}$ and GilBERT $\mathrm{L}$ for $1 \mathrm{M}$ steps, with learning rate as $5 e-5$ and $1 e-5$, batch size as 384 and 256 .

3.5.2 Fine-tuning. During each epoch, we randomly select $50 \%$ training data to be complete image-text pairs, and the other $50 \%$ to be modality-missing ones, whose either text or image data is dropped with half chance.

Given the transformer (T), text-to-image generator $(\mathbf{G})$, discriminators (D) and image-to-text captioner (C) of the proposed GilBERT, the goal is to optimize the following minimax objective:

$$
\min _{\theta_{G}, \theta_{T}, \theta_{C}} \max _{\theta_{D}} L_{g e n}(G, C, T)+\alpha L_{X E}(C)+\beta L_{d i s}(G, C, D),
$$

where $L_{g e n}$ stands for generator loss, which is the sum of Masked Token Modeling (MTM) loss and text-to-image feature synthesizing; $L_{\text {dis }}$ stands for discriminator loss, including both Image Text Matching (ITM) loss and two feature-wise losses in Section 3.4.2; $L_{X E}$ is the cross-entropy loss of image-to-text captioning model. 


\begin{tabular}{|c|c|c|c|c|c|c|c|c|c|c|c|c|c|}
\hline \multirow{3}{*}{ Method } & \multirow{3}{*}{ Size } & \multicolumn{6}{|c|}{$1 \mathrm{~K}$ Test Set } & \multicolumn{6}{|c|}{$5 \mathrm{~K}$ Test Set } \\
\hline & & \multicolumn{3}{|c|}{ Image Query } & \multicolumn{3}{|c|}{ Text Query } & \multicolumn{3}{|c|}{ Image Query } & \multicolumn{3}{|c|}{ Text Query } \\
\hline & & $\mathrm{R} @ 1$ & $\mathrm{R} @ 5$ & R@10 & R@1 & R@5 & R@10 & $\mathrm{R} @ 1$ & $\mathrm{R} @ 5$ & R@10 & $\mathrm{R} @ 1$ & R@5 & $\mathrm{R} @ 10$ \\
\hline DVSA [24] & - & 38.4 & 69.9 & 80.5 & 27.4 & 60.2 & 74.8 & - & - & - & - & - & - \\
\hline VSE $++[10]$ & - & 64.7 & - & 95.9 & 52.0 & - & 92.0 & 41.3 & - & 81.2 & 30.3 & - & 72.4 \\
\hline VLP [68] & - & 65.6 & 89.8 & 95.5 & 47.1 & 79.9 & 90.0 & 41.2 & 70.5 & 81.1 & 25.3 & 53.4 & 66.4 \\
\hline CAMP [58] & - & 72.3 & 94.8 & 98.3 & 58.5 & 87.9 & 95.0 & 50.1 & 82.1 & 89.7 & 39.0 & 68.9 & 80.2 \\
\hline SCAN [27] & - & 72.7 & 94.8 & 98.4 & 58.8 & 88.4 & 94.8 & 50.4 & 82.2 & 90.0 & 38.6 & 69.3 & 80.4 \\
\hline SCG [45] & - & 76.6 & 96.3 & 99.2 & 61.4 & 88.9 & 95.1 & 56.6 & 84.5 & 92.0 & 39.2 & 68.0 & 81.3 \\
\hline PFAN [57] & - & 76.5 & 96.3 & 99.0 & 61.6 & 89.6 & 95.2 & - & - & - & - & - & - \\
\hline VSRN [29] & - & - & - & - & - & - & - & 53.0 & 81.1 & 89.4 & 40.5 & 70.6 & 81.1 \\
\hline ViLBERT [36] & B & - & - & - & 58.2 & 84.9 & 91.5 & - & - & - & - & - & - \\
\hline 12-in-1 [37] & B & - & - & - & 65.2 & 91.0 & 96.2 & - & - & - & - & - & - \\
\hline Unicoder-VL [28] & B & 84.3 & 97.3 & 99.3 & 69.7 & 93.5 & 97.2 & 62.3 & 87.1 & 92.8 & 46.7 & 76.0 & 85.3 \\
\hline UNITER [5] & B & - & - & - & - & - & - & 63.3 & 87.0 & 93.1 & 48.4 & 76.7 & 85.9 \\
\hline UNITER [5] & $\mathrm{L}$ & - & - & - & - & - & - & 66.6 & 89.4 & 94.3 & 51.7 & 78.4 & 86.9 \\
\hline Oscar [34] & B & 88.4 & 99.1 & 99.8 & 75.7 & 95.2 & 98.3 & 70.0 & 91.1 & 95.5 & 54.0 & 80.8 & 88.5 \\
\hline Oscar [34] & $\mathrm{L}$ & 89.8 & 98.8 & 99.7 & 78.2 & 95.8 & 98.3 & 73.5 & 92.2 & 96.0 & 57.5 & 82.8 & 89.8 \\
\hline GilBERT & B & 89.0 & 99.5 & 99.6 & 77.0 & 95.9 & 99.1 & 72.1 & 92.3 & 96.0 & 54.7 & 80.8 & 89.1 \\
\hline GilBERT & $\mathrm{L}$ & 90.5 & 99.4 & 99.3 & 79.6 & 97.0 & 99.2 & 75.6 & 93.8 & 97.1 & 58.3 & 84.0 & 91.1 \\
\hline
\end{tabular}

Table 2: Cross-modal retrieval. The database items are either images or texts, not image-text pairs.

Depending on which modality is missed, there are three kinds of samples, i.e., image only, text only, and image-text pair, which corresponds to three simplified forms of Equation 6, that is:

Image-text Pair. In this case, we do not need to involve the generative parts, i.e., text-to-image synthesizing and image-to-text captioning. Therefore, $L_{g e n}$ and $L_{d i s}$ are essentially $L_{M T M}$ and $L_{I T M}$, and $L_{X E}$ can be removed. The optimization objectives can be simplified into:

$$
\min _{\theta_{T}, \theta_{D}} L_{M T M}(G, C, T)+\beta L_{I T M}(D) .
$$

Text Only. In case that image data is dropped, we take apart the image-to-text captioning model and rewrite Equation 6 as:

$$
\min _{\theta_{G}, \theta_{T}} \max _{\theta_{D}} L_{g e n}(G, C, T)+\beta L_{d i s}(G, C, D),
$$

which is commonly seen in GAN [38]. Thus, we follow [38] to optimize Equation 8 in a two-step manner. During the first step, we update $\mathrm{G}$ and $\mathrm{T}$ to minimize Equation 8, while keep the $\mathrm{D}$ fixed. During the second step, we fix $\mathrm{G}$ and $\mathrm{T}$, update $\mathrm{D}$ to maximize Equation 8.

Image Only. In this case, we can fix the parameters of text-toimage synthesizing model. Thus, we replace $L_{\text {dis }}$ with $L_{I T M}$ and rewrite Equation 6 as:

$$
\min _{\theta_{C}, \theta_{T}} \max _{\theta_{D}} L_{M T M}(G, C, T)+\alpha L_{X E}(C)+\beta L_{I T M}(G, C, D),
$$

which can be updated in a two-step iterative manner similar to Equation 8.

\section{EXPERIMENTS}

To ensure that the image region features have the same input embedding size as BERT, we transform the position-sensitive region features using a linear projection. The sequence length of discrete tokens and region features are 35 and 50, respectively. Following [46], we pre-train our GilBERT on an union of four existing $\mathrm{V}+\mathrm{L}$ datasets, including COCO [35], Conceptual Captions [44], SBU captions [39], flicker30k [60], GQA [23], etc. We denote two variants of our models as GilBERT $\mathrm{B}$ and GilBERT $_{\mathrm{L}}$, for their different initialization with BERT base $(H=768)$ and large $(H=1024)$, respectively, where $H$ is the hidden size. We set $\alpha$ and $\beta$ as 0.5 and 1.0 in our experiments, mainly aiming to balance the scale of the three losses. We observe that the experimental results not sensitive to minor changes of $\alpha$ and $\beta$.

We adapt the pre-trained GilBERT model to five downstream V-L tasks, including Image-Text Retrieval, Image Captioning, Novel Object Captioning, Visual Question Answering and Natural Language Visual Reasoning for Real, with focuses on the Image-Text Retrieval.

\subsection{Application to Image-Text Retrieval}

Image-Text Retrieval significantly relies on a comprehensive representation of image-text pair. As discussed in Section 2.2, we group image-text retrieval into cross-modal retrieval and multi-modal retrieval, depending on whether database items are single-modal image/text, or image-text pairs. Though we emphasize more on multi-modal retrieval in this work, we start our evaluations by 


\begin{tabular}{l|ccc}
\hline Method & VSRN [29] & Oscar $_{\mathrm{B}}$ [34] & GilBERT $_{\mathrm{B}}$ \\
\hline Inference time & 0.84 second & 12 minutes & 0.92 second \\
\hline
\end{tabular}

Table 3: Retrieval time for text query on $5 \mathrm{~K}$ Test Set.

firstly experimenting on cross-modal retrieval that have be extensively studied in previous works. By comparing with existing works, the advantages of our method can be clearly demonstrated, then we move to multi-modal retrieval which is less explored in literature.

We conduct experiments on COCO dataset [35], which contains $82 \mathrm{~K}$ training images, $5 \mathrm{~K}$ validation images and $5 \mathrm{~K}$ test images. Each image has 5 captions. During training, we randomly drop its image or caption for each input pair, and train our generative models to complete the fragmentary pair. Meanwhile, we formulate the fine-tuning of transformer as a binary classification problem., i.e., the final representation of [CLS] is used as the input to the classifier to predict whether the given pair is fake or not.

4.1.1 Cross-modal retrieval. There are two sub-tasks in cross-modal retrieval: image retrieval and text retrieval, depending on which modality is used as the retrieved target. For both input query and database items, we generate the missing modality based on the available one, and use the final representation of [CLS] as feature representation.

The retrieval accuracy are shown in Table 2. We group the compared methods into two categories, i.e., injective embedding methods from DVSA [24] to VSRN [29], VLP methods from ViLBERT [36] to Oscar [34]. We have the following observations: 1). VLP methods significantly outperform traditional injective embeddings such as PFAN [57] or VSRN [29]. For example, the Recall@1 of Oscar [34] is higher than VSRN [29] by at least 10 for both image and text retrieval. 2). Compared with existing VLP methods, our GilBERT can improve the retrieval accuracy in all cases, except Recall@10 for image query on $1 \mathrm{~K}$ Test Set.

We compared the retrieval efficiency in Table 3 with an injective embedding method VSRN [29] and a VLP method Oscar [34]. Both VSRN [29] and GilBERT take $\sim 0.9$ seconds per query for VBDC on the $5 \mathrm{~K}$ test set, the slight time difference is caused by feature extraction of the incoming query. In contrast, Oscar $B$ [34] takes up to 12 minutes! Other VLP methods are expected to consume similar time to Oscar $_{B}$ [34] as they all require an exhaustive inference over the entire database. The gap in efficiency will further increase with techniques like hash $[15,17,20,56]$ or quantization $[18,55,61,66]$.

4.1.2 Multi-modal retrieval. The setting of multi-modal retrieval is designed analogue to practical e-commerce scenarios, i.e., the query is text inputted by user, while database items are image-text pairs. Existing injective embedding or VLP methods do not fit well in this setting, since they are incapable to effectively utilize the text data of database items. In contrast, our GilBERT simultaneously handles image and text data and produce a joint representation, facilitates high-accuracy computation-friendly information retrieval.

We conduct the experiments on COCO dataset, whose images are associated with five captions. We randomly select one of the five captions as query, and pair the remaining four captions with their images as database items. The representations of database

\begin{tabular}{lc|ccc|ccc}
\hline \multirow{2}{*}{ Method } & \multirow{2}{*}{ Size } & \multicolumn{3}{|c}{ 1K Test Set } & \multicolumn{3}{c}{ 5K Test Set } \\
& & R@1 & R@5 & R@10 & R@1 & R@5 & R@10 \\
\hline Sota IE & - & 61.6 & 89.6 & 95.2 & 40.5 & 70.6 & 81.1 \\
\hline Bert & B & 74.9 & 94.3 & 97.2 & 53.3 & 80.0 & 87.5 \\
Bert & L & 77.4 & 94.9 & 97.5 & 57.2 & 82.3 & 89.3 \\
\hline Sota VLP & B & 75.7 & 95.2 & 98.3 & 54.0 & 80.8 & 88.5 \\
Sota VLP & L & 78.2 & 95.8 & 98.3 & 57.5 & 82.8 & 89.8 \\
\hline GilBERT & B & $\mathbf{7 8 . 4}$ & $\mathbf{9 6 . 7}$ & $\mathbf{9 9 . 3}$ & $\mathbf{5 6 . 0}$ & $\mathbf{8 2 . 2}$ & $\mathbf{8 9 . 8}$ \\
GilBERT & L & $\mathbf{8 1 . 0}$ & $\mathbf{9 8 . 1}$ & $\mathbf{9 9 . 7}$ & $\mathbf{5 9 . 3}$ & $\mathbf{8 5 . 8}$ & $\mathbf{9 1 . 7}$ \\
\hline
\end{tabular}

Table 4: Multi-modal retrieval. Sota IE and VLP stands for the state-of-the-art injective embedding and visionlanguage pretraining methods. Sota IE are from PFAN [57] and VSRN [29], Sota VLP is exactly Oscar [34].

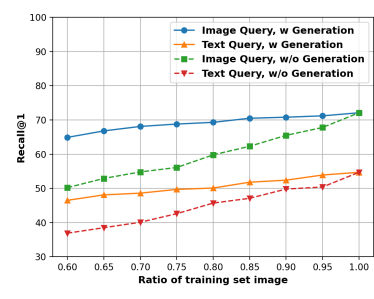

(a) Image data missing.

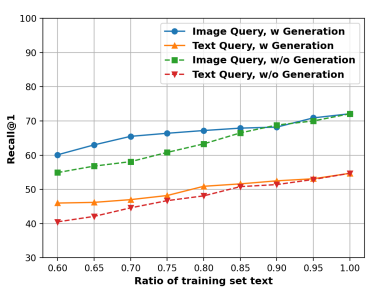

(b) Text data missing.
Figure 4: The utilization to incomplete data in training set. We intentionally drop part of image or text data in the training set, and train GilBERT with or without using the nonparallel image/text data. The solid and dashed lines denote that the non-parallel data is used in training or not. We observe that GilBERT can well harness the non-parallel data for achieving better retrieval recall.

items are directly extracted by the visual-linguistic transformer of GilBERT, while the representation of query requires the involvement of both text-to-image synthesizing and transformer. We note that the application of GilBERT to multi-modal retrieval shares the spirit of query expansion [7].

The experimental results are presented in Table 4 . We select the state-of-the-art results of injective embedding and VLP method for comparisons. We also compare with purely text-based retrieval to demonstrate the necessity of taking visual information into consideration. The superior performances validate the advantages of the proposed GilBERT.

\subsection{Data Augmentation}

Paired data are costly to collect and hard to scale up as they require either manual annotations $[4,25]$, or extensive screening. For example, it is reported in [44] that from 5 billion images gathered over the Internet, only 3 million have paired high-quality captions that are eventually included in the Conceptual Caption dataset [44]. Therefore, some pioneer work has attempted to perform visual-linguistic pre-training on non-parallel image and text corpus [31]. 


\begin{tabular}{|c|c|c|c|c|c|c|c|c|c|c|c|c|c|}
\hline \multirow{3}{*}{ Method } & \multirow{3}{*}{ Size } & \multicolumn{6}{|c|}{$1 \mathrm{~K}$ Test Set } & \multicolumn{6}{|c|}{$5 \mathrm{~K}$ Test Set } \\
\hline & & \multicolumn{3}{|c|}{ Image Query } & \multicolumn{3}{|c|}{ Text Query } & \multicolumn{3}{|c|}{ Image Query } & \multicolumn{3}{|c|}{ Text Query } \\
\hline & & R@1 & R@5 & R@10 & R@1 & R@5 & R@10 & R@1 & $\mathrm{R} @ 5$ & R@10 & R@1 & R@5 & R@10 \\
\hline GilBERT & B & 89.0 & 99.5 & 99.6 & 77.0 & 95.9 & 99.1 & 72.1 & 92.3 & 96.0 & 54.7 & 80.8 & 89.1 \\
\hline GilBERT + Zero Padding & B & 77.2 & 88.4 & 89.7 & 65.9 & 86.8 & 88.2 & 41.8 & 62.0 & 64.4 & 44.2 & 66.0 & 67.6 \\
\hline GilBERT + Exhaustive Matching & B & 89.2 & 99.6 & 99.7 & 77.3 & 96.3 & 99.4 & 72.4 & 92.4 & 96.3 & 54.9 & 81.0 & 89.3 \\
\hline GilBERT + Image-Text Alignment & B & 84.5 & 94.6 & 94.9 & 72.0 & 91.0 & 94.7 & 67.2 & 88.2 & 91.0 & 52.1 & 77.0 & 84.9 \\
\hline GilBERT + Captions by LSTM [59] & B & 88.6 & 99.2 & 99.5 & 76.2 & 95.8 & 98.8 & 71.8 & 92.0 & 95.3 & 54.2 & 80.3 & 88.6 \\
\hline GilBERT + Images by ObjGAN [33] & $\mathrm{B}$ & 86.0 & 97.0 & 97.2 & 73.4 & 92.5 & 95.8 & 69.2 & 89.7 & 93.2 & 51.7 & 77.4 & 85.6 \\
\hline Oscar [34] & $\mathrm{B}$ & 88.4 & 99.1 & 99.8 & 75.7 & 95.2 & 98.3 & 70.0 & 91.1 & 95.5 & 54.0 & 80.8 & 88.5 \\
\hline Oscar [34] + Captions by GilBERT & $\mathrm{B}$ & 88.1 & 98.9 & 99.6 & 75.3 & 95.0 & 98.1 & 69.8 & 90.9 & 95.0 & 53.8 & 80.4 & 88.1 \\
\hline Oscar [34] + Captions by LSTM [59] & $\mathrm{B}$ & 88.1 & 98.8 & 99.4 & 75.0 & 94.6 & 97.8 & 69.4 & 90.8 & 95.0 & 53.3 & 80.2 & 87.9 \\
\hline Oscar [34] + Images by ObjGAN [33] & B & 85.9 & 96.4 & 96.8 & 72.5 & 92.0 & 95.4 & 67.1 & 88.5 & 92.3 & 53.2 & 77.6 & 85.8 \\
\hline
\end{tabular}

Table 5: Ablation studies on cross-modal retrieval. The database items are either images or texts, not image-text pairs.

Though our GilBERT is mainly designed for handling incomplete pairs in inference stage, a natural question to ask is: are the generative components capable to complete the training data? It will be very beneficial if the single-modal image or text corpus can be used in visual-linguistic pre-training in future. We briefly investigate this problem by intentionally simulating such a scenario. Specifically, when we fine-tune the pre-trained VLP models on COCO dataset, we start with $60 \%$ of image-text pairs, and gradually adding the other $40 \%$ image or text to the training set. We illustrate the change of retrieval accuracy in Figure 4. We observe that the performance of GilBERT increases w.r.t. image or text data, demonstrating that our method is capable to learn from non-parallel image and text corpus.

\subsection{Ablation Studies}

4.3.1 Why GilBERT excels? In this section, we experimentally investigate why GilBERT outperforms other VLP methods such as Oscar [34]. We conjecture the possible reasons could be: 1). The generated image-text pairs are more suitable for retrieval applications. 2). The VBDC is not only faster than exhaustive matching, but also leads to better retrieval accuracy. 3). The Image-Text Matching of generated samples provides better supervision than Image-Text Alignment of other VLP methods.

To validate the conjectures above, we design the following experiments: 1). We replace image-to-text captioner and text-to-imagesynthesizer as separately trained LSTM [59] and ObjGAN [33], and monitor the changes of retrieval accuracy to see whether the generated contents matter. We also compare with Zero Padding as a baseline. 2). We perform retrieval using trained GilBERT in the exhaustive matching manner, then compare the differences between exhaustive matching and VBDC. 3). We add the Image-Text Alignment loss to our GilBERT, and train it by additional randomly constructed negative pairs.

The experimental results are presented in Table 5. We observe that: 1). The captions generated by LSTM [59] result in slight performance drop, while the gap cased by images generated by ObjGAN [33] is more significant. The reason could be that current

\begin{tabular}{ll|ccc|ccc}
\hline & & \multicolumn{3}{|c|}{ Image Query } & \multicolumn{3}{c}{ Text Query } \\
& & R@1 & R@5 & R@10 & R@1 & R@5 & R@10 \\
\hline \multirow{4}{*}{ 1K Test Set } & FW Only & 83.8 & 95.7 & 96.1 & 73.1 & 91.5 & 94.9 \\
& PF Only & 81.7 & 96.3 & 96.7 & 74.0 & 92.2 & 95.8 \\
& Both & $\mathbf{8 9 . 0}$ & $\mathbf{9 9 . 5}$ & $\mathbf{9 9 . 6}$ & $\mathbf{7 7 . 0}$ & $\mathbf{9 5 . 9}$ & $\mathbf{9 9 . 1}$ \\
\hline \multirow{5}{*}{ 5K Test Set } & FW Only & 68.3 & 89.0 & 90.4 & 50.6 & 75.9 & 86.6 \\
& PF Only & 67.9 & 90.2 & 91.6 & 51.0 & 76.4 & 88.3 \\
& Both & $\mathbf{7 2 . 1}$ & $\mathbf{9 2 . 3}$ & $\mathbf{9 6 . 0}$ & $\mathbf{5 4 . 7}$ & $\mathbf{8 0 . 8}$ & $\mathbf{8 9 . 1}$ \\
\hline
\end{tabular}

Table 6: Ablations on the discriminator. FW and PF stand for feature-wise discriminator and permutation-free discriminator, respectively.

image synthesizing methods are not mature enough and often produce artifacts. 2). The retrieval performances of GilBERT is even slightly better after switching to exhaustive matching from VBDC. 3). The drops caused by negative image-text samples are significant. This is consistent with the observations in VL-BERT [47], i.e., the involvement of image-text alignment loss introduces massive unmatched image and caption pairs as negative samples, which hamper the training and hurt retrieval performances.

In summary, the merits of modality completing by generation are two-fold. On the one hand, the generated image/text completes the fragmentary pairs for unified representation extraction, which facilitates efficient VBDC in testing stage. On the other hand, the high-quality generation leads to accurate discrimination through the generative adversarial training, without suffering from massive negative samples. The discriminator plays a critical role in learning representations of image-text pairs, and leads to our superior retrieval performances.

4.3.2 The Variants of Discriminator. We experimentally study the design of discriminator in visual feature synthesizing. As shown in Table 6, our feature-wise discriminator and permutation-free discriminator work together to provide both local and global scale supervision to visual feature synthesizing, validating the rationale of our design. 


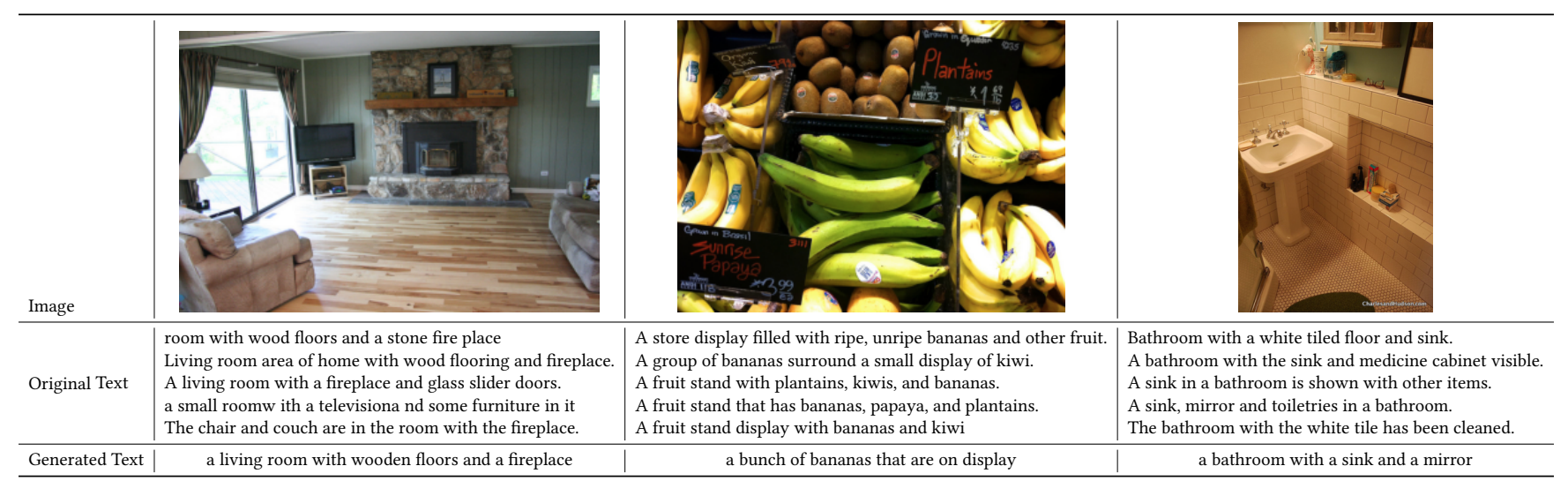

Table 7: Image-to-text captioning.

\begin{tabular}{|c|c|c|c|c|c|c|c|c|c|}
\hline \multirow{2}{*}{ Task } & \multirow{2}{*}{ Size } & \multicolumn{4}{|c|}{ Image Captioning } & \multicolumn{2}{|c|}{ NoCaps } & \multirow{2}{*}{$\begin{array}{c}\text { VQA } \\
\text { test-std }\end{array}$} & \multirow{2}{*}{$\begin{array}{l}\text { NLVR2 } \\
\text { test-P }\end{array}$} \\
\hline & & B@4 & $M$ & C & S & $\mathrm{C}$ & S & & \\
\hline VLP [68] & - & 36.5 & 28.4 & 117.7 & 21.3 & - & - & 72.54 & 74.50 \\
\hline UpDown [22] & - & - & - & - & - & 74.3 & 11.2 & - & - \\
\hline Oscar [34] & B & 40.5 & 29.7 & 137.6 & 22.8 & 78.8 & 11.7 & 73.44 & 78.36 \\
\hline Oscar [34] & $\mathrm{L}$ & 41.7 & 30.6 & 140.0 & 24.5 & 80.9 & 11.3 & 73.82 & 80.37 \\
\hline GilBERT & B & 37.4 & 28.5 & 129.4 & 21.5 & 75.7 & 10.4 & 71.45 & 76.62 \\
\hline GilBERT & $\mathrm{L}$ & 38.2 & 29.3 & 133.7 & 22.6 & 77.7 & 10.9 & 72.91 & 78.54 \\
\hline
\end{tabular}

Table 8: Overall results on four visual-linguistic tasks.

\begin{tabular}{ll|ccc|ccc}
\hline & & \multicolumn{3}{|c|}{ Image Query } & \multicolumn{3}{c}{ Text Query } \\
& & R@1 & R@5 & R@10 & R@1 & R@5 & R@10 \\
\hline \multirow{2}{*}{ 1K Test Set } & separate training & 87.8 & 98.7 & 99.1 & 74.1 & 91.5 & 94.9 \\
& joint training & $\mathbf{8 9 . 0}$ & $\mathbf{9 9 . 5}$ & $\mathbf{9 9 . 6}$ & $\mathbf{7 7 . 0}$ & $\mathbf{9 5 . 9}$ & $\mathbf{9 9 . 1}$ \\
\hline \multirow{2}{*}{$5 \mathrm{~K}$ Test Set } & separate training & 69.6 & 90.2 & 95.3 & 51.4 & 78.9 & 88.0 \\
& joint training & $\mathbf{7 2 . 1}$ & $\mathbf{9 2 . 3}$ & $\mathbf{9 6 . 0}$ & $\mathbf{5 4 . 7}$ & $\mathbf{8 0 . 8}$ & $\mathbf{8 9 . 1}$ \\
\hline
\end{tabular}

Table 9: Joint training v.s. separate training.

4.3.3 Joint Training. It is also doable to separately train visuallinguistic transformer, image-to-text captioning model and text-toimage synthesizing model, but we found that joint optimization can improve the experimental performances as shown in Table 9.

4.3.4 Qualitative study of generative models. Although generation is not the focus of this work, the outputs of our generative models are still worth investigating as they demonstrate the quality of generation. The quality of text-to-image synthesizing is hard to assess, while the results of image-to-text captioning is easy to visualize, as presented in Table 7.

\subsection{Application to other four $\mathrm{V}+\mathrm{L}$ tasks}

We conduct further experiments on other four visual-linguistic downstream tasks: Image Captioning [35], Novel Object Captioning (NoCaps) [1, 26], Visual Question Answering (VQA) [11] and Natural Language Visual Reasoning for Real (NLVR2) [48]. Unlike image-text retrieval, these four tasks do not suffer from modality incompleteness or massive negative samples caused by random sampling, thus the generative models are not updated, i.e., our GilBERT degrades to a common VLP approach with only the visual-linguistic transformer trained. As shown in Table 8, our GilBERT performs on par with several existing VLP methods, but not as good as the state-of-the-art one Oscar [46] due to the lack of object tags [46].

\section{CONCLUSION}

In this work, we point out the shortcoming of existing VLP methods in retrieval applications, and tackle the problem by proposing a generative visual-linguistic pre-training approach. The proposed GilBERT harnesses generative models to complete fragmentary image-text pairs, meanwhile extract generic image-text representations by visual-linguistic transformers. Thanks to the help of generative modeling and removal of negative samples, we achieve excellent image-text retrieval performances in terms of both accuracy and efficiency. Moreover, our method demonstrates improved performances with non-parallel image or text added, which opens the possibility of training visual-linguistic transformers with singlemodal corpus. Extensive experiments demonstrate the advantages of GilBERT against prior state-of-the-arts methods.

\section{ACKNOWLEDGMENTS}

The authors appreciate Kaisheng Yao and Haoyuan Peng for their generous help! The leading author Weixiang Hong cannot agree more that Haoyuan Peng is the most handsome guy in Room 505. 


\section{REFERENCES}

[1] Agrawal, H., Desai, K., Wang, Y., Chen, X., Jain, R., Johnson, M., Batra, D., PARIKh, D., LeE, S., AND Anderson, P. nocaps: novel object captioning at scale. In ICCV (2019).

[2] Alberti, C., Ling, J., Collins, M., And Reitter, D. Fusion of detected objects in text for visual question answering. In EMNLP-I7CNLP (2019).

[3] Babenko, A., and Lempitsky, V. The inverted multi-index. TPAMI (2014).

[4] Chen, X., Fang, H., Lin, T.-Y., Vedantam, R., Gupta, S., Dollár, P., And Zitnick, C. L. Microsoft coco captions: Data collection and evaluation server. arXiv preprint arXiv:1504.00325 (2015)

[5] Chen, Y.-C., Li, L., Yu, L., Kholy, A. E., Ahmed, F., Gan, Z., Cheng, Y., And LiU, J. Uniter: Learning universal image-text representations. In ECCV (2020).

[6] Cheng, J., Wu, F., Tian, Y., WANG, L., AND TAO, D. Rifegan: Rich feature generation for text-to-image synthesis from prior knowledge. In CVPR (2020).

[7] Chum, O., Philbin, J., Sivic, J., Isard, M., and Zisserman, A. Total recall: Automatic query expansion with a generative feature model for object retrieval. In ICCV (2007).

[8] Deng, J., Dong, W., Socher, R., Li, L.-J., Li, K., And Fei-Fei, L. Imagenet: A large-scale hierarchical image database. In CVPR (2009).

[9] Devlin, J., Chang, M.-W., Lee, K., And Toutanova, K. Bert: Pre-training of deep bidirectional transformers for language understanding. In NAACL HLT (2019).

[10] Faghri, F., Fleet, D. J., Kiros, J. R., ANd Fidler, S. Vse++: Improving visualsemantic embeddings with hard negatives. arXiv:1707.05612 (2017).

[11] Goyal, Y., Khot, T., Summers-Stay, D., Batra, D., And Parikh, D. Making the $\mathrm{v}$ in vqa matter: Elevating the role of image understanding in visual question answering. In CVPR (2017).

[12] Gu, J., CAI, J., Joty, S. R., Niu, L., AND WANG, G. Look, imagine and match: Improving textual-visual cross-modal retrieval with generative models. In CVPR (2018).

[13] Gu, J., CAI, J., WANG, G., AND Chen, T. Stack-captioning: Coarse-to-fine learning for image captioning. In $A A A I$ (2018)

[14] Hong, S., YANG, D., CHOI, J., AND LEE, H. Inferring semantic layout for hierarchical text-to-image synthesis. In CVPR (2018)

[15] Hong, W., Chang, Y.-T., Qin, H., Hung, W.-C., Tsai, Y.-H., And Yang, M.-H Image hashing via linear discriminant learning. In WACV (2020).

[16] Hong, W., Meng, J., And Yuan, J. Distributed composite quantization. In $A A A I$ (2018)

[17] Hong, W., Meng, J., And Yuan, J. Tensorized projection for high-dimensional binary embedding. In $A A A I(2018)$.

[18] Hong, W., Tang, X., Meng, J., And Yuan, J. Asymmetric mapping quantization for nearest neighbor search. TPAMI (2019).

[19] Hong, W., WANG, Z., YANG, M., AND YuAN, J. Conditional generative adversarial network for structured domain adaptation. In CVPR (2018).

[20] Hong, W., AND YuAn, J. Fried binary embedding: From high-dimensional visual features to high-dimensional binary codes. TIP (2018).

[21] Hong, W., Yuan, J., and Das Bhattacharjee, S. Fried binary embedding for high-dimensional visual features. In CVPR (2017).

[22] Huang, L., WANG, W., Chen, J., AND WeI, X.-Y. Attention on attention for image captioning. In ICCV (2019).

[23] Hudson, D. A., And Manning, C. D. Gqa: A new dataset for real-world visual reasoning and compositional question answering. In CVPR (2019).

[24] Karpathy, A., And Fei-Fei, L. Deep visual-semantic alignments for generating image descriptions. In CVPR (2015).

[25] Krishna, R., Zhu, Y., Groth, O., Johnson, J., Hata, K., Kravitz, J., Chen, S., Kalantidis, Y., Li, L.-J., Shamma, D. A., ET AL. Visual genome: Connecting language and vision using crowdsourced dense image annotations. IFCV (2017)

[26] Kuznetsova, A., Rom, H., Alldrin, N., Uijlings, J., Krasin, I., Pont-Tuset, J. Kamali, S., Popov, S., Malloci, M., Duerig, T., et Al. The open images dataset v4: Unified image classification, object detection, and visual relationship detection at scale. arXiv:1811.00982 (2018)

[27] Lee, K.-H., Chen, X., Hua, G., Hu, H., And He, X. Stacked cross attention for image-text matching. In ECCV (2018).

[28] Li, G., Duan, N., FAnG, Y., Jiang, D., ANd Zhou, M. Unicoder-vl: A universal encoder for vision and language by cross-modal pre-training. arXiv preprint arXiv:1908.06066 (2019).

[29] LI, K., ZHANG, Y., LI, K., LI, Y., AND Fu, Y. Visual semantic reasoning for image-text matching. In ICCV (2019).

[30] Li, L. H., Yatskar, M., Yin, D., Hsieh, C.-J., and Chang, K.-W. Visualbert: A simple and performant baseline for vision and language. arXiv:1908.03557 (2019).

[31] Li, L. H., You, H., Wang, Z., Zareian, A., Chang, S.-F., And Chang, K.-W Weakly-supervised visualbert: Pre-training without parallel images and captions. arXiv:2010.12831 (2020).

[32] Li, W., Zhang, P., Zhang, L., HuAng, Q., He, X., Lyu, S., ANd GaO, J. Object-driven text-to-image synthesis via adversarial training. In CVPR (2019).

[33] Li, W., Zhang, P., Zhang, L., Huang, Q., He, X., Lyu, S., AND GaO, J. Object-driven text-to-image synthesis via adversarial training. In CVPR (2019).

[34] Li, X., Yin, X., Li, C., Zhang, P., Hu, X., Zhang, L., Wang, L., Hu, H., Dong, L.,
WEI, F., ET AL. Oscar: Object-semantics aligned pre-training for vision-language tasks. In ECCV (2020).

[35] Lin, T.-Y., Maire, M., Belongie, S., Hays, J., Perona, P., Ramanan, D., Dollár, P., AND Zitnick, C. L. Microsoft coco: Common objects in context. In ECCV (2014).

[36] Lu, J., Batra, D., Parikh, D., ANd Lee, S. Vilbert: Pretraining task-agnostic visiolinguistic representations for vision-and-language tasks. In NIPS (2019).

[37] Lu, J., Goswami, V., Rohrbach, M., Parikh, D., AND Lee, S. 12-in-1: Multi-task vision and language representation learning. In CVPR (2020).

[38] Mirza, M., AND Osindero, S. Conditional generative adversarial nets. arXiv:1411.1784 (2014).

[39] Ordonez, V., Kulkarni, G., And Berg, T. L. Im2text: Describing images using 1 million captioned photographs. In NIPS (2011).

[40] QI, C. R., Su, H., Mo, K., AND Guibas, L. J. Pointnet: Deep learning on point sets for 3d classification and segmentation. In CVPR (2017)

[41] Reed, S., Akata, Z., Yan, X., Logeswaran, L., Schiele, B., And Lee, H. Generative adversarial text to image synthesis. In ICML (2016).

[42] Ren, S., He, K., Girshick, R., AND Sun, J. Faster r-cnn: Towards real-time object detection with region proposal networks. In NIPS (2015)

[43] Ren, Z., WANG, X., ZhANG, N., Lv, X., AND LI, L.-J. Deep reinforcement learningbased image captioning with embedding reward. In CVPR (2017).

[44] Sharma, P., Ding, N., Goodman, S., And Soricut, R. Conceptual captions: A cleaned, hypernymed, image alt-text dataset for automatic image captioning. In $A C L$ (2018).

[45] Socher, R., AND Fei-FeI, L. Connecting modalities: Semi-supervised segmentation and annotation of images using unaligned text corpora. In CVPR (2010).

[46] Song, Y., AND SolEymani, M. Oscar: Object-semantics aligned pre-training for vision-language tasks. In ECCV (2020).

[47] Su, W., Zhu, X., CAO, Y., LI, B., Lu, L., Wei, F., AND DAI, J. Vl-bert: Pre-training of generic visual-linguistic representations. In ICLR (2019).

[48] Suhr, A., Zhou, S., Zhang, A., Zhang, I., Bai, H., ANd Artzi, Y. A corpus for reasoning about natural language grounded in photographs. arXiv:1811.00491 (2018).

[49] Sun, C., Baradel, F., Murphy, K., And Schmid, C. Contrastive bidirectional transformer for temporal representation learning. arXiv:1906.05743 (2019).

[50] Sun, C., Myers, A., Vondrick, C., Murphy, K., ANd Schmid, C. Videobert: A joint model for video and language representation learning. In ICCV (2019).

[51] TAN, H., AND BANSAL, M. Lxmert: Learning cross-modality encoder representations from transformers. In EMNLP-IFCNLP (2019).

[52] Vaswani, A., Shazeer, N., Parmar, N., Uszkoreit, J., Jones, L., Gomez, A. N., KaISER, Ł., AND Polosukhin, I. Attention is all you need. In NIPS (2017).

[53] Vinyals, O., Bengio, S., And Kudlur, M. Order matters: Sequence to sequence

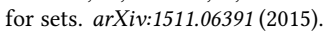

[54] Vinyals, O., Toshev, A., Bengio, S., And Erhan, D. Show and tell: A neural image caption generator. In CVPR (2015).

[55] Wang, Z., Hong, W., TAN, Y.-P., AND YuAn, J. Pruning 3d filters for accelerating 3d convnets. TMM (2019).

[56] WANG, Z., Hong, W., AND YuAn, J. Deep reinforcement learning with label embedding reward for supervised image hashing. arXiv preprint arXiv:2008.03973 (2020).

[57] Wang, Z., LiU, X., Li, H., Sheng, L., Yan, J., Wang, X., And Shao, J. Camp: Cross-modal adaptive message passing for text-image retrieval. In ICCV (2019).

[58] Wu, Q., Shen, C., Liu, L., Dick, A., And Van Den Hengel, A. What value do explicit high level concepts have in vision to language problems? In CVPR (2016).

[59] Xingjian, S., Chen, Z., Wang, H., Yeung, D.-Y., Wong, W.-K., and Woo, W.-c. Convolutional lstm network: A machine learning approach for precipitation nowcasting. In NIPS (2015).

[60] Young, P., Lai, A., Hodosh, M., And Hockenmaier, J. From image descriptions to visual denotations: New similarity metrics for semantic inference over event descriptions. Transactions of the Association for Computational Linguistics (2014).

[61] Yu, T., Meng, J., FAng, C., Jin, H., ANd Yuan, J. Product quantization network for fast visual search. IFCV (2020).

[62] Yu, T., WANG, Z., AND YUAN, J. Compressive quantization for fast object instance search in videos. In ICCV (2017)

[63] Yu, T., Wu, Y., Bhattacharjee, S., and Yuan, J. Efficient object instance search using fuzzy objects matching. In AAAI (2017).

[64] Yu, T., Wu, Y., AND YuAN, J. Hope: Hierarchical object prototype encoding for efficient object instance search in videos. In CVPR (2017).

[65] Yu, T., YANG, Y., Li, Y., LiU, L., FeI, H., AND Li, P. Heterogeneous attention network for effective and efficient cross-modal retrieval. In SIGIR (2021).

[66] Yu, T., Yuan, J., FAng, C., AND Jin, H. Product quantization network for fast image retrieval. In ECCV (2018)

[67] Zhang, H., Xu, T., Li, H., Zhang, S., Wang, X., Huang, X., and Metaxas, D. N. Stackgan: Text to photo-realistic image synthesis with stacked generative adversarial networks. In ICCV (2017).

[68] Zhou, L., Palangi, H., Zhang, L., Hu, H., Corso, J. J., and Gao, J. Unified vision-language pre-training for image captioning and vqa. In $A A A I(2020)$. 